\title{
Is the far border of the Local Void expanding? ${ }^{\star, \star \star}$
}

\author{
I. Iwata ${ }^{1, \star \star \star}$ and P. Chamaraux ${ }^{2}$ \\ 1 Okayama Astrophysical Observatory, National Astronomical Observatory of Japan, Honjo, Kamogata, Asakuchi, \\ Okayama 719-0232, Japan \\ e-mail: ikuru.iwata@nao.ac.jp \\ 2 Observatoire de Paris - Meudon, GEPI, 92195 Meudon Cedex, France
}

Received 10 February 2011 / Accepted 14 April 2011

\section{ABSTRACT}

\begin{abstract}
Context. According to models of evolution in the hierarchical structure formation scenarios, voids of galaxies are expected to expand. The Local Void (LV) is the closest large void, and it provides a unique opportunity to test observationally such an expansion. It has been found that the Local Group, which is on the border of the LV, is running away from the void center at $\sim 260 \mathrm{~km} \mathrm{~s}^{-1}$.

Aims. In this study we investigate the motion of the galaxies at the far-side border of the LV to examine the presence of a possible expansion.

Methods. We selected late-type, edge-on spiral galaxies with radial velocities between $3000 \mathrm{~km} \mathrm{~s}^{-1}$ and $5000 \mathrm{~km} \mathrm{~s}^{-1}$, and carried out $\mathrm{HI} 21 \mathrm{~cm}$ line and $H$-band imaging observations. The near-infrared Tully-Fisher relation was calibrated with a large sample of galaxies and carefully corrected for Malmquist bias. It was used to compute the distances and the peculiar velocities of the LV sample galaxies. Among the 36 sample LV galaxies with good quality HI line width measurements, only 15 galaxies were selected for measuring their distances and peculiar velocities, in order to avoid the effect of Malmquist bias.

Results. The average peculiar velocity of these 15 galaxies is found to be $-419_{-251}^{+208} \mathrm{~km} \mathrm{~s}^{-1}$, which is not significantly different from zero.

Conclusions. Due to the intrinsically large scatter of Tully-Fisher relation, we cannot conclude whether there is a systematic motion against the center of the LV for the galaxies at the far-side boundary of the void. However, our result is consistent with the hypothesis that those galaxies at the far-side boundary have an average velocity of $\sim 260 \mathrm{~km} \mathrm{~s}^{-1}$ equivalent to what is found at the position of the Local Group.
\end{abstract}

Key words. galaxies: distances and redshifts - large-scale structure of Universe

\section{Introduction}

Deep extended galaxy surveys have shown that the large-scale distribution of galaxies consists in matter concentrations, such as clusters, filaments, and walls, and also in vast regions devoid of galaxies, i.e. the voids. These voids occupy the largest volumes in the Universe, according to Ceccarelli et al. (2006), and the radii of the voids those authors find in the $2 \mathrm{dF}$ galaxy redshift survey range from 5 to $25 h^{-1} \mathrm{Mpc}\left(h=H_{0} / 100\right)$.

Voids are expected to expand, since galaxies undergo a gravitational pull at their borders from the objects located outside them. Sheth \& van de Weygaert (2004) have developed a model of the evolution of voids, which indeed leads to an expansion of the surviving voids at the present time. On the other hand, Ceccarelli et al. (2006) have modeled the velocity field around

\footnotetext{
* Based on data taken at Nançay radiotelescope operated by Observatoire de Paris, CNRS and Université d'Orléans, Infrared Survey Facility (IRSF) which is operated by Nagoya university under the cooperation of South African Astronomical Observatory, Kyoto University, and National Astronomical Observatory of Japan.

$\star \star$ This publication makes use of data products from the Two Micron All Sky Survey, which is a joint project of the University of Massachusetts and the Infrared Processing and Analysis Center/ California Institute of Technology, funded by the National Aeronautics and Space Administration and the National Science Foundation.

$\star \star \star$ Present address: Subaru Telescope, National Astronomical Observatory of Japan, 650 North A'ohoku Place, Hilo, HI 96720, USA.
}

the voids found in their study, and show that the expansion velocity is maximum at the edge of the voids and is proportional to the void radius, for instance reaching $210 \mathrm{~km} \mathrm{~s}^{-1}$ for a void with a radius of $12.5 h^{-1} \mathrm{Mpc}$.

It is also possible to directly measure the expansion velocities at the edge of a peculiar void, namely the Local Void (LV), taking advantage of its being very close to us. The LV was discovered by Tully \& Fisher (1987) from their survey of galaxies with redshift lower than $3000 \mathrm{~km} \mathrm{~s}^{-1}$. Its structure has been investigated by Nakanishi et al. (1997) from a visual search of IRAS galaxies behind the Milky Way, since the major part of this void is at galactic latitude $|b|<15^{\circ}$. They localize its center at $\ell=60^{\circ}, b=-15^{\circ}, c z=2500 \mathrm{~km} \mathrm{~s}^{-1}$, and they find that it extends to $c z=5000 \mathrm{~km} \mathrm{~s}^{-1}$. On the other hand, the Local Group and neighboring galaxies are located at the boundary of the LV, as shown by Tully et al. (2008).

By accurate measurements of distances of 200 galaxies within $10 \mathrm{Mpc}$ carried out with the Hubble Space Telescope, Tully et al. (2008) find that the Local Group and its neighboring galaxies are running away from the center of the LV with a velocity of $259 \mathrm{~km} \mathrm{~s}^{-1}$. This proves the expansion of the LV at our location and also solves the problem of the so-called "Local Velocity Anomaly" appearing in the motion of the LG relative to the CMB (Faber \& Burstein 1988; Burstein 2000).

In the present study, we intend to determine the peculiar velocities of galaxies located at the edge of the LV opposite to us $\left(c z \sim 3000-5000 \mathrm{~km} \mathrm{~s}^{-1}\right)$ in order to check whether the LV also 
undergoes an observable expansion in that region. The peculiar velocities are computed from the distances of the galaxies measured by means of the near-infrared Tully-Fisher relation (hereafter IRTFR) using near-infrared and HI 21-cm observations.

The organization of the paper is the following. Section 2 presents the sample of the LV galaxies observed. And then the IR and HI measurements are described and the data of interest are given. In Sect. 3, the IRTFR in $H$-band is determined from a calibration sample and corrected for Malmquist bias. In Sect. 4 we compute the distances of the LV galaxies from the IRTFR and derive their peculiar velocities after correction of the observed radial velocities from infall into some nearby mass concentrations. Concluding remarks are given in Sect. 5.

\section{Observations}

\subsection{The sample selection}

We selected uniquely spiral galaxies for the HI and IR observations, since the TF relation is only valid for them. These objects were chosen as located at the edge of the LV opposite to us and slightly beyond, at galactic coordinates: $30^{\circ}<l<70^{\circ},|b|<20^{\circ}$ (i.e., around the North Supergalactic Pole), and with recession velocities $c z<5000 \mathrm{~km} \mathrm{~s}^{-1}$ (see the maps by Nakanishi et al. 1997). In addition to the galaxies in the literature (most of them are listed in the UGC catalog, Nilson 1973), we executed the redshift measurement observations for some galaxies that were discovered in a systematic optical search by Roman et al. (2000). The observations were done in July and October 2000 using the New Cassegrain Spectrograph attached to the $188 \mathrm{~cm}$ reflector of the Okayama Astrophysical Observatory, National Astronomical Observatory of Japan. In Table 1 we list these new radial velocities. Among these galaxies with new radial velocity measurements, those at $c z<5000 \mathrm{~km} \mathrm{~s}^{-1}$ were added to the sample, except UGC 11417, which does not satisfy the limit of the axial ratio (less than 0.71 ; see below).

Use of the TF relation needs the determination of the maximal rotational velocity $V_{\mathrm{m}}$ of each galaxy, and $V_{\mathrm{m}}$ is obtained from the width $W$ of the $21-\mathrm{cm}$ HI profile by $V_{\mathrm{m}} \sim W /(2 \sin i)$, where $i$ is the inclination of the galaxy. To obtain accurate $V_{\mathrm{m}}$, we only kept galaxies with $i>45 \mathrm{deg}$, i.e., galaxies with axial ratios in the three-band coadded images in the Two Micron All Sky Survey (2MASS; Skrutskie et al. 2006), named "sup-ba" in the extended source catalog (XSC) less than 0.71 (assuming an intrinsic axis ratio of 0.2 for a spiral galaxy viewed edge-on).

Moreover, we need good $S / N$ in the HI profiles to obtain good HI widths, hence accurate distances by the TF relation. Since the HI fluxes of the spiral galaxies are proportional to the square of their apparent diameters, such an accuracy is obtained by selecting only galaxies with sufficiently large apparent diameters. Taking into account the sensitivity of the Nançay radiotelescope, we kept mainly galaxies having an extinction-corrected major axis larger than one arcmin.

Finally, our observational sample comprises 50 galaxies with measured redshifts. In Figs. 1 and 2 we show the spatial distribution of these galaxies. It is shown in these figures that the sample galaxies well represent the population of the far-side boundary of the LV.

\section{2. $\mathrm{HI} 21-\mathrm{cm}$ line observations}

The 21-cm line observations of the LV galaxies were carried out with the Nançay radiotelescope. This instrument is a meridian one, with a half-power beam width at $21-\mathrm{cm}$ of
Table 1. List of galaxies with new heliocentric radial velocity $V_{\mathrm{h}}$ measurements in $\mathrm{km} \mathrm{s}^{-1}$ in the catalog of Roman et al. (2000).

\begin{tabular}{ccccc}
\hline \hline CGMW5 ID & Designation & RA(2000) & $\operatorname{Dec}(2000)$ & $V_{\mathrm{h}}$ \\
\hline CGMW5-00817 & CGCG 084-014 & $18: 04: 14.7$ & $+09: 20: 06$ & 6293.5 \\
CGMW5-03387 & - & $18: 24: 42.9$ & $+12: 20: 23$ & 5564.3 \\
CGMW5-05003 & - & $18: 33: 28.9$ & $+22: 29: 17$ & 3864.3 \\
CGMW5-05171 & - & $18: 34: 17.8$ & $+22: 48: 27$ & 4196.3 \\
CGMW5-05908 & - & $18: 37: 16.1$ & $+31: 46: 21$ & 4599.3 \\
CGMW5-06653 & - & $18: 41: 19.7$ & $+24: 07: 10$ & 3968.8 \\
CGMW5-06881 & - & $18: 42: 59.7$ & $+21: 36: 18$ & 4379.9 \\
CGMW5-07342 & - & $18: 45: 13.5$ & $+31: 57: 35$ & 7981.9 \\
CGMW5-10456 & UGC 11417 & $19: 14: 22.1$ & $+29: 58: 34$ & $3958.6^{a}$ \\
\hline
\end{tabular}

Notes. ${ }^{(a)}$ The radial velocity of UGC 11417 has been reported to be $3970 \mathrm{~km} \mathrm{~s}^{-1}$ by Springob et al. (2005).

$3.6 \operatorname{arcmin}(\mathrm{E}-\mathrm{W}) \times 22 \operatorname{arcmin}(\mathrm{N}-\mathrm{S})$ at zero declination (nearly the same value for our galaxies, declinations of which are between $+10 \mathrm{deg}$ and $+25 \mathrm{deg}$ ). The system temperature is $35 \mathrm{~K}$. We used a bandwidth of $25 \mathrm{MHz}$ covered by 2048 channels of the spectrometer, resulting in a velocity resolution of $2.6 \mathrm{~km} \mathrm{~s}^{-1}$. The observations were performed in an on-off mode, and the integration time on each galaxy generally ranged from 1 to $2 \mathrm{~h}$.

Forty-three galaxies of our sample were observed between the years 2000 and 2004, leading to 30 detections, one possible detection and 12 non-detections. The line profiles of the detected galaxies were reduced using a hanning and boxcar smoothing, leading to a final velocity resolution of $2.6 \times 4=$ $10.4 \mathrm{~km} \mathrm{~s}^{-1}$. The line profiles of the detected galaxies and of the possibly detected one (UGC 11198) are shown in Fig. 3, after the hanning and boxcar smoothing and the subtraction of the polynomial fitted baseline. The parameters of interest derived from the profile were obtained, namely the widths $W_{20}$ and $W_{50}$ of the profile at $20 \%$ and $50 \%$ of the peak intensity, the heliocentric velocity $V_{\mathrm{h}}$, and the HI flux $F_{\mathrm{H}}$. All these quantities are given in Table 2, with other data for the galaxies that are useful for the present study. For five galaxies (UGC 11254, UGC 11285, CGMW5-05908, UGC 11323, and UGC 11426), observations were disturbed by the Sun. However, the line widths can be measured correctly. On the other hand, the profiles of IRAS $18340+1016$ and NGC 6930 are confused, and their line widths cannot be measured, so these two galaxies are not listed in Table 2 .

The list of the 12 undetected galaxies is the following: CGCG 114-006, CGCG 172-027, CGCG 201-043, CGMW505619, FGC 2187, NGC 6586, NGC 6641, NGC 6658, UGC 11301, UGC 11353, UGC 11368, and UGC 11369.

Description of Table 2: Col. (1) Name of the galaxy. (2) Equatorial coordinates $\alpha, \delta$ (2000). (3) Galactic coordinates $\ell, b$. (4) Heliocentric radial velocity $V_{\mathrm{h}}$ in $\mathrm{km} \mathrm{s}^{-1}$. (5) Major and minor axes $a_{\mathrm{c}}$ and $b_{\mathrm{c}}$ in arcmin measured at the isophotal level of $25 \mathrm{mag} / \mathrm{arcsec}^{2}$ in the $B$-band, and corrected for inclination and for galactic extinction (those data come from Hyperleda database). (6) Position angle in degrees, from UGC or 2MASS XSC when the galaxy is not included in the UGC. (7) Galactic dust attenuation in $V$-band, from the map by Schlegel et al. (1998). (8) and (9) Widths $W_{20}$ and $W_{50}$ of the HI line in $\mathrm{km} \mathrm{s}^{-1}$ at $20 \%$ and $50 \%$ of its maximum height, respectively, uncorrected for velocity resolution, with their 

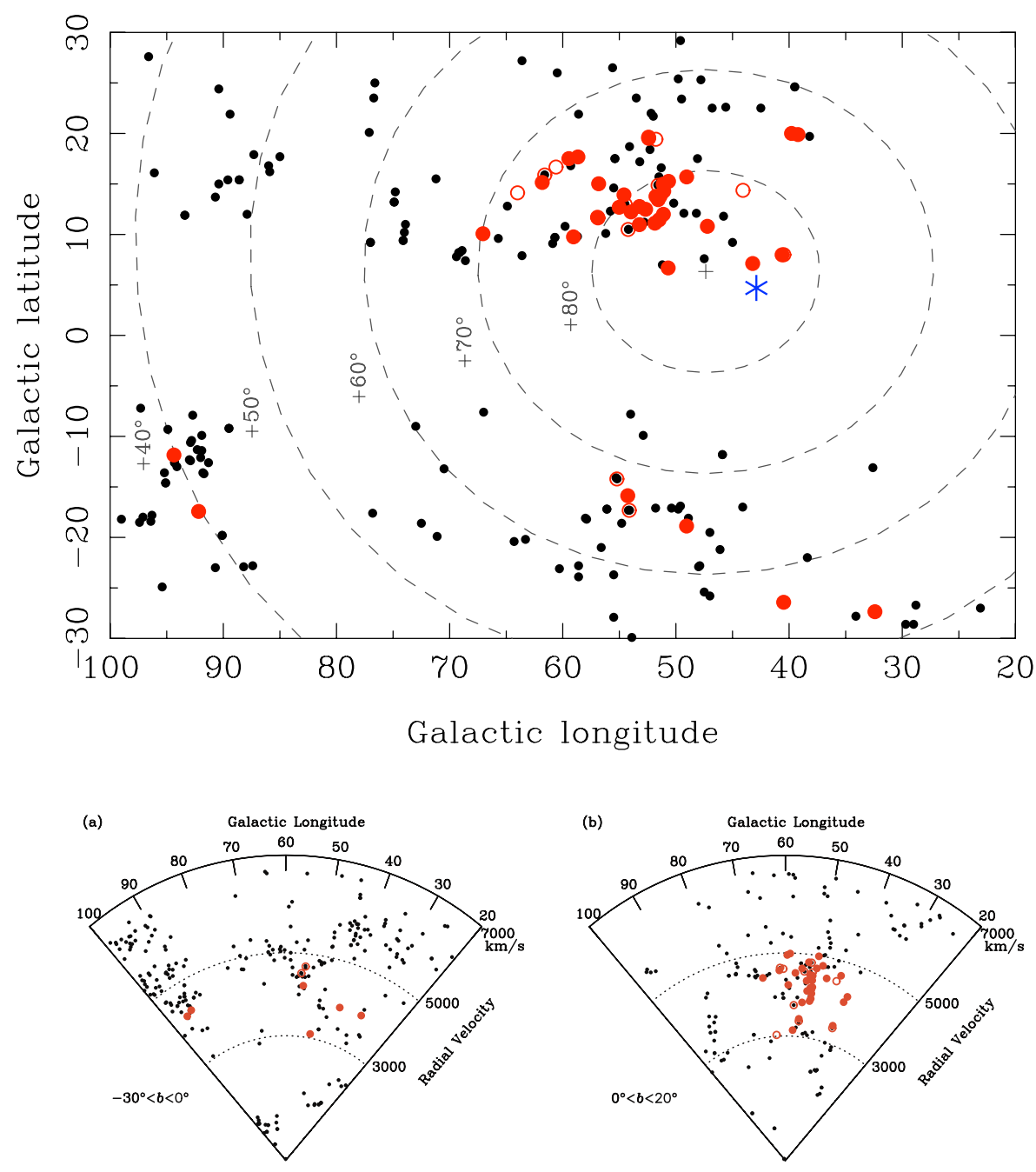

Fig. 1. Spatial distribution of the Local Void sample galaxies in galactic coordinates. Small filled circles represent galaxies in the IRAS PSCz catalog (Saunders et al. 2000) with radial velocity between $3000 \mathrm{~km} \mathrm{~s}^{-1}$ and $5000 \mathrm{~km} \mathrm{~s}^{-1}$. Larger circles are the Local Void sample galaxies in this study (50 galaxies, see Sect. 2.1). Filled circles are for the 36 galaxies included in the final sample (see Sect. 2.3), while open circles are those not in the final sample. Dashed lines indicate latitudes in the supergalactic coordinates, and the cross shows the position of the North supergalactic pole. The position opposite to the Local Velocity Anomaly defined by Burstein (2000) is indicated by a blue asterisk.

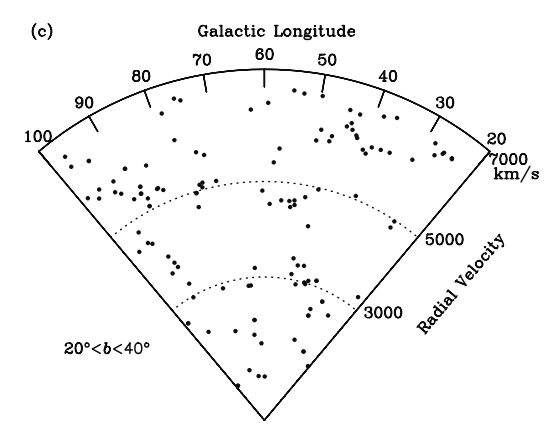

Fig. 2. Distribution of the Local Void sample galaxies in the galactic longitude - radial velocity planes. a)-c) display the galaxies with galactic latitude $-30^{\circ}<b<0^{\circ}, 0^{\circ}<b<20^{\circ}$ and $20^{\circ}<b<40^{\circ}$, respectively. The meaning of symbols are the same as in Fig. 1: filled circles represent galaxies in the IRAS PSCz catalog (Saunders et al. 2000) with radial velocities. Larger circles are the 50 Local Void sample galaxies in this study. Larger filled circles are for the 36 galaxies included in the final sample, and open circles are those not in the final sample.

uncertainties (after Fouqué et al. 1990). (10) Measured HI flux $F_{\mathrm{H}}$, in $\mathrm{Jy} \times \mathrm{km} \mathrm{s}^{-1}$, with its uncertainty:

$e_{F_{\mathrm{H}}}=5 F_{\mathrm{H}}^{0.5}(S / N)^{-1} R^{0.5} h^{0.5}$

where $S / N$ is at the point of the profile of maximum intensity, $R$ the resolution in $\mathrm{km} \mathrm{s}^{-1}$, and $h$ the peak intensity of the HI line (after Fouqué et al. 1990). (11) HI flux $F_{\mathrm{H}, \mathrm{c}}$ corrected for beam attenuation; $f_{0}$ is the correction factor such that $F_{\mathrm{H}, \mathrm{c}}=f_{0} F_{\mathrm{H}}$. $f_{0}$ is given by

$f_{0}=\sqrt{1+\left(\frac{D_{\mathrm{H}_{E W}}}{3.6}\right)^{2}}$

where $D_{\mathrm{H}}$ is the HI diameter of the galaxy, within which half of the HI mass is contained; $D_{\mathrm{H}_{E W}}$ is the projection of this diameter in the east-west direction, expressed in arcmin. One has $D_{\mathrm{H}}=a_{\mathrm{c}}$ (after Hewitt et al. 1983). If $\theta$ is the position angle of the galaxy, its east-west corrected diameter is given by

$a_{E W}=\sqrt{a_{\mathrm{c}}^{2} \sin ^{2} \theta+b_{\mathrm{c}}^{2} \cos ^{2} \theta}$.

Corrections for beam attenuation are small, only $3 \%$ on an average, except for NGC 6674 and IRAS 18575+1845, where they reach $20-30 \%$. (12) Notes.

\subsection{The final sample of galaxies measured in the HI line and selected for use in the IR TF relation}

First we add to our initial sample of 30 detected galaxies 18 other galaxies located on the opposite border of the LV and measured elsewhere in the HI line (three of them, namely FCG 2187, UGC 11301 and UGC 11369 have not been detected by us). Thus we have a sample of 48 galaxies measured in the HI line at our disposal. One can note that 17 among our 30 detected galaxies have also been detected elsewhere (thus only 13 are newly measured by us).

In order to use the IRTFR in the best conditions, we need to have the best profile width $W_{20}$. Thus we suppress all the cases of inaccurate $W$, of possible confusion, and of too narrow a profile corresponding to dwarf galaxies for which the TFR does not work correctly. There are 12 such galaxies, namely: UGC 11150, UGC 11253, UGC 11371, UGC 11552, IRAS 18340+1016, NGC 6930 (profile confusion), CGMW5-06653, CGCG 143-017, UGC 11333, UGC 11369 (too narrow or asymmetrical profile), CGMW5-05908 ( $W_{20}$ not accurate enough), and FCG 2187 (uncertain detection).

On the other hand, for galaxies measured by us and elsewhere as well, we have examined the two profiles obtained. If they were of equivalent quality, we took the average of the 

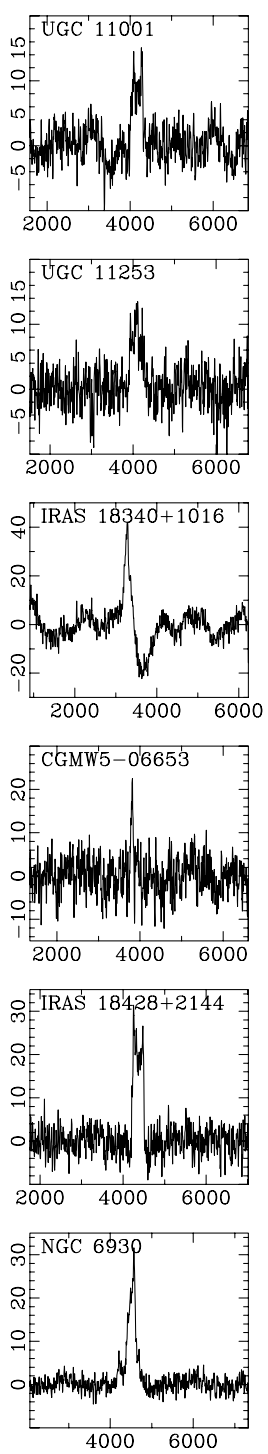
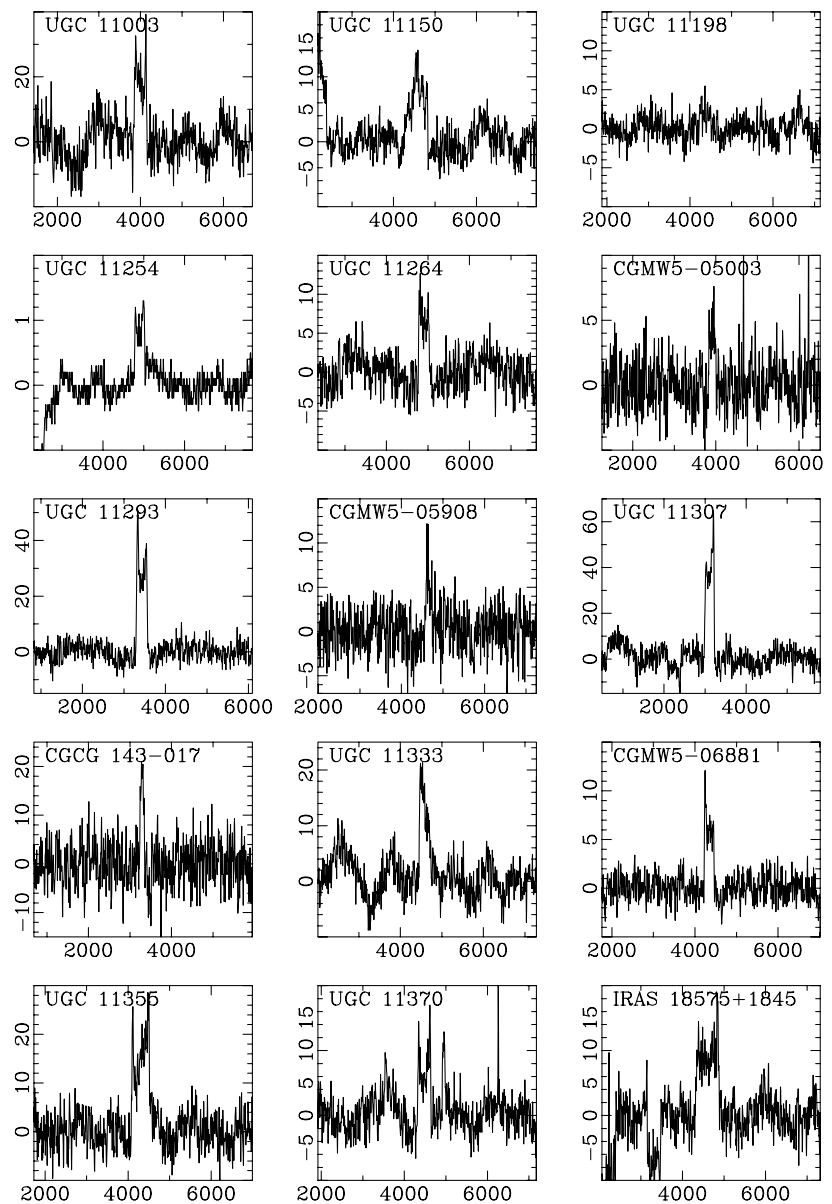
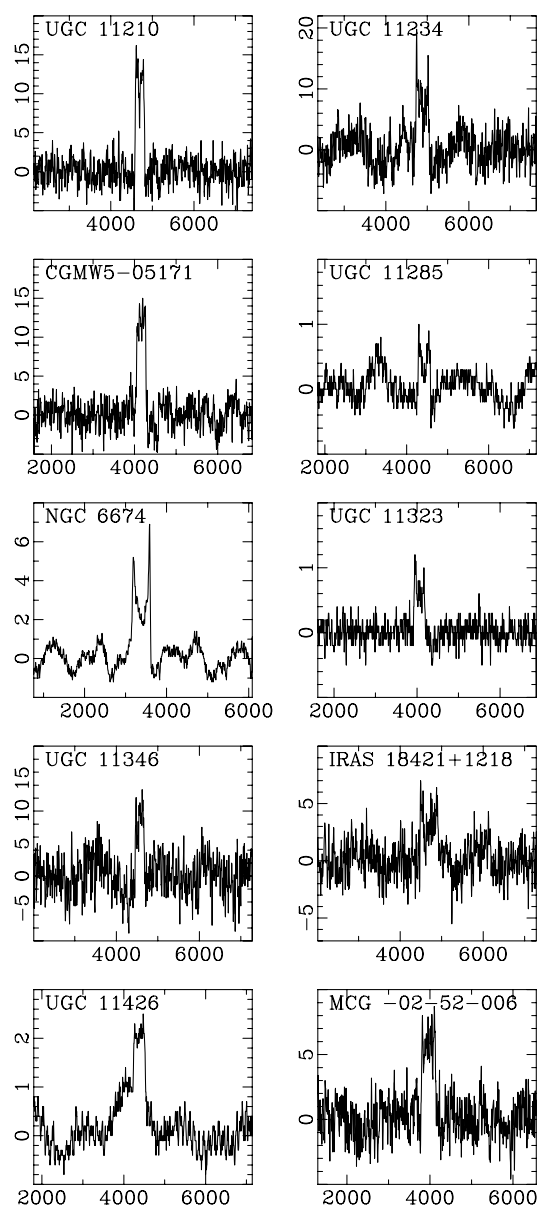

Fig. 3. The HI $21 \mathrm{~cm}$ line profiles of the 30 Local Void galaxies detected with Nançay radiotelescope and of the possibly detected one (UGC 11198). The horizontal axis is the radial velocity in $\mathrm{km} \mathrm{s}^{-1}$ and the vertical axis is the flux density in mJy. The hanning and boxcar smoothing are applied and the subtraction of the polynomial fitted baseline is made.

two values for $W_{20}$ (after correction for velocity resolution). If one profile was much better than the other one, its $W_{20}$ value was preferred.

Our final sample comprises 36 galaxies, namely, 19 galaxies for which our $W_{20}$ or average of ours and others have been taken, four galaxies measured by us and others and for which the other $W_{20}$ have been preferred, and 13 galaxies measured only elsewhere. The final values of $W_{20}$ were corrected for velocity resolution $R$ by $W_{20}^{\text {cor }}=W_{20}-0.55 R$ (Bottinelli et al. 1990). For our own measurements, the correction is $-4.7 \mathrm{~km} \mathrm{~s}^{-1}$. These corrected $W_{20}$ values are used to compute the widths corrected for inclination and redshift: $W_{20}^{\mathrm{c}}=W_{20}^{\text {cor }} /(\sin i(1+z))$ used in IRTFR. The $\log W_{20}^{\text {c }}$ values are presented in Table 3.

Description of Table 3: (1) name of the galaxy. (2) Equatorial coordinates $\alpha, \delta$ (2000). (3) Galactic coordinates $\ell, b$. (4) Heliocentric radial velocity $V_{\mathrm{h}}$ in $\mathrm{km} \mathrm{s}^{-1}$. (5) Inclination of the galaxy in degrees. (6) $H$-band $20 \mathrm{mag} / \operatorname{arcsec}^{2}$ isophotal elliptical magnitude and its error. (7) $H$-band magnitude corrected for galactic and internal extinctions. (8) Source of $H$-band photometry. "UH88" and "IRSF" stand for photometry based on our own observations with the UH 2.2 m telescope and the IRSF $1.4 \mathrm{~m}$ telescope, respectively. "2MAS" stands for data taken from the 2MASS XSC. (9) Logarithm of the width of the HI line at $20 \%$ of its maximum height, corrected for velocity resolution, inclination, and redshift, in $\mathrm{km} \mathrm{s}^{-1}$. (10) Logarithm of the maximum of the rotation velocity in $\mathrm{km} \mathrm{s}^{-1}$, derived from $\log W_{\mathrm{c}}$. See text for details. (11) Source of HI line width data. "Nançay": our own measurements. "Springob": Springob et al. (2005). "LEDA": the on-line galaxy database HyperLEDA (Paturel et al. 2003a). "Mean": the average value of measurements by our observations at Nançay, HyperLEDA, and Springob et al. (2005) (when available).

\subsection{Near-IR (H-band) observations and data reduction}

Near-infrared ( $H$-band) imaging observations were carried out using two facilities. One is the Quick Near-Infrared Camera (QUIRC) equipped to the University of Hawaii $2.2 \mathrm{~m}$ telescope (UH88) at Mauna Kea, Hawaii, and the other one is the near-infrared Simultaneous Infrared Imager for Unbiased Survey (SIRIUS: Nagashima et al. 1999; Nagayama et al. 2003) on board the Infrared Survey Facility (IRSF) telescope in the 
I. Iwata and P. Chamaraux: Is the far border of the Local Void expanding?

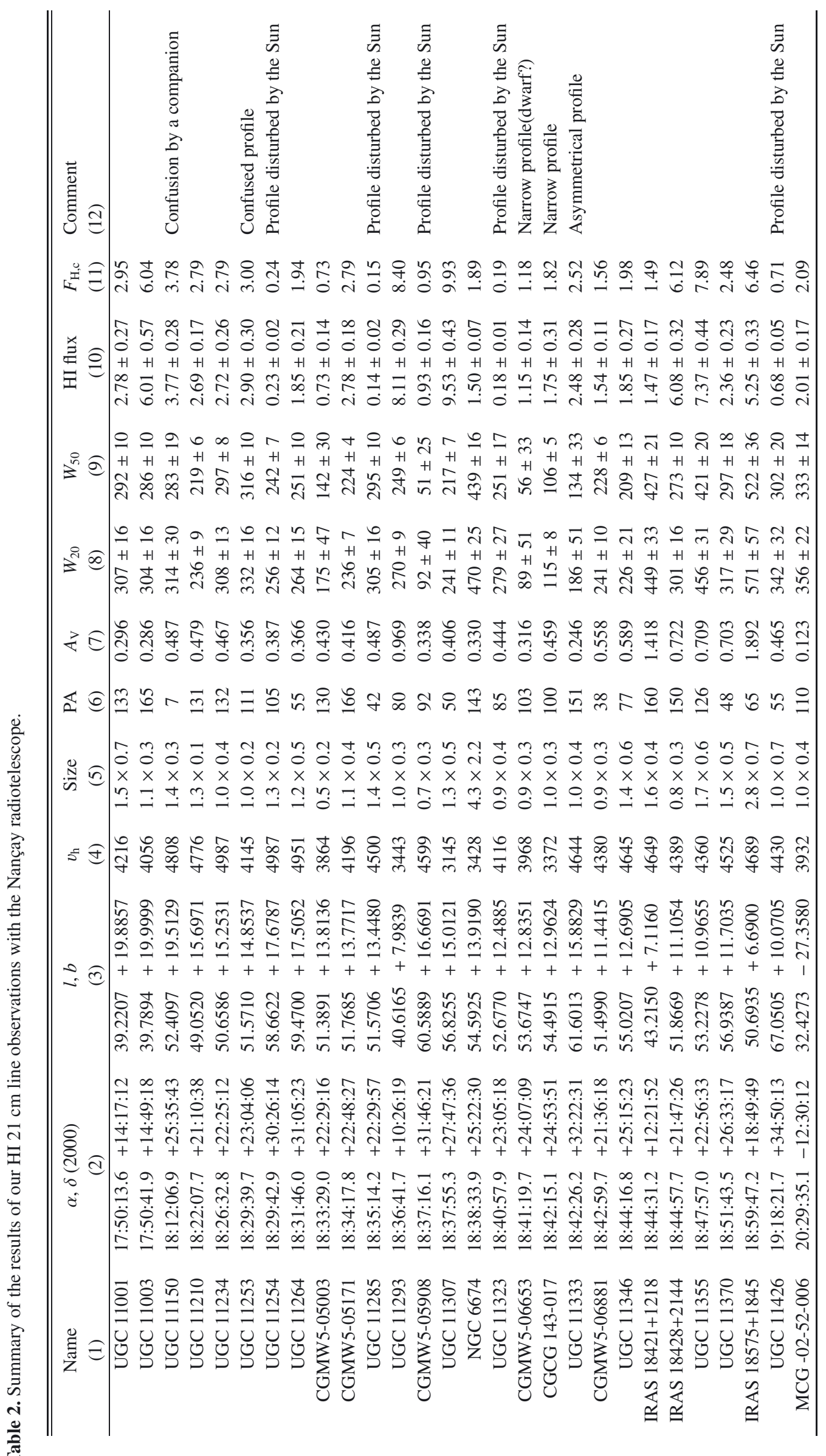




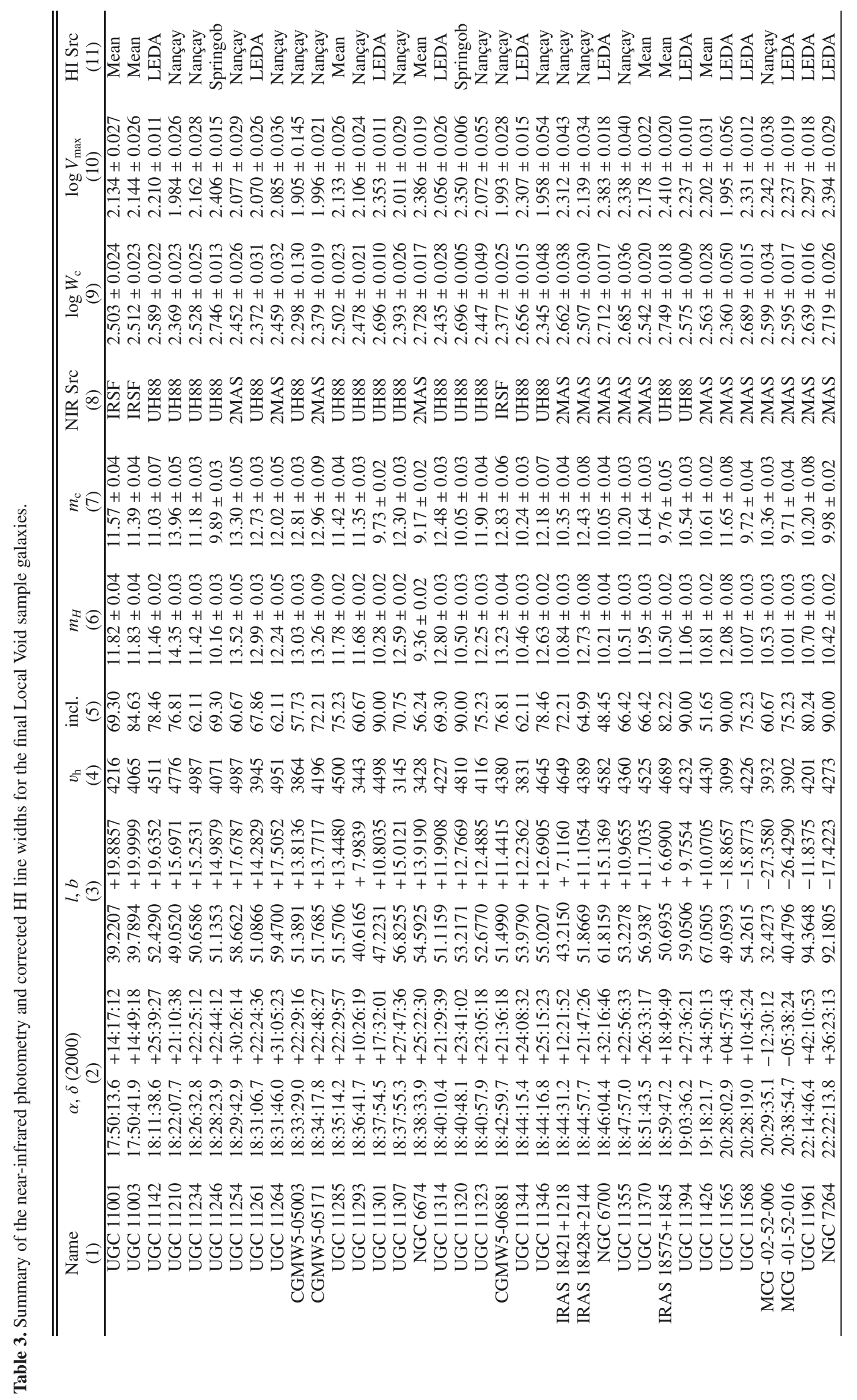


South African Astronomical Observatory (SAAO) at Sutherland, South Africa.

Observations with UH88/QUIRC were carried out in 2001 July 7-9 and August 4-5 (UT). The condition was mostly photometric throughout observing dates. QUIRC has a HAWAII $1024 \times 1024 \mathrm{HgCdTe}$ array with pixel scale of $0.19^{\prime \prime} /$ pixel, yielding a field-of-view of $193^{\prime \prime} \times 193^{\prime \prime}$. Total on-source integration times range from $600 \mathrm{~s}$ to $1200 \mathrm{~s}$, depending on the apparent surface brightness of objects.

Observations with IRSF/SIRIUS were made during 2003 March 31-April 4 and 2004 March 14-22. Although the SIRIUS camera has a capability of obtaining $J, H$, and $K$ s images simultaneously, in the present analysis only $H$-band images are used for the $H$-band Tully-Fisher relation. The field of view and the pixel scale of SIRIUS are $7 ! 7 \times 7 ! 7$ and 0.45 , respectively. Total on-source integration times are $900 \mathrm{~s}$ for UGC 11001 and CGMW5-06881, and 1200 s for UGC 11003.

Basic data reduction, including dark subtraction, flatfielding, image alignment, and stacking, was done in a standard way using IRAF. Since the LV region is close to the Galactic plane, it is crowded with foreground stars. It is quite important to remove these stars before executing the photometry of target galaxies, for precise measurement of their apparent magnitude. For faint stars we did PSF fitting using Moffat profile for each star and subtracted them from reduced images. For bright stars their profiles are saturated, and we could not execute profile fitting. In that case we removed these stars by interpolation of counts from surrounding pixels using an IRAF task "IMEDIT". After the removal of foreground stars, isophotal ellipses with $H=20 \mathrm{mag} / \operatorname{arcsec}^{2}$ were defined for each galaxy, and we calculated counts within the ellipse. Photometric zero points were derived for each night using near-infrared standard stars.

For 15 objects in our sample we could not obtain our own $H$-band imaging data. For these objects we used data in the 2MASS XSC. We used $20 \mathrm{mag} / \mathrm{arcsec}^{2}$ isophotal elliptical aperture magnitude ( $h \_m \_i 20$ e) in the catalog. With the galaxies for which we obtained imaging data with UH88 and IRSF, we checked the consistency of the photometry between ours and the 2MASS XSC. For galaxies with relatively fewer foreground stars in the aperture (which can disturb the automated photometric procedure adopted in the 2MASS XSC), we found that the difference between our isophotal magnitudes and those in 2MASS XSC is less than $0.1 \mathrm{mag}$ in most cases. In Table 3 we list the results of $H$-band photometry for the final sample galaxies.

\section{Near-infrared Tully-Fisher relation}

We use the Tully-Fisher relation (TFR) to compute the distances of the LV sample galaxies. We first proceed here to determine the TFR in the $H$-band. Generally speaking, the Tully-Fisher relation (TFR) is an empirical linear relationship between the logarithm of the maximum rotational velocity $V_{\mathrm{m}}$ of any spiral galaxy and its absolute magnitude $M$, namely,

$M=a \log V_{\mathrm{m}}+b$,

where $a$ and $b$ are constant quantities in a given system of magnitudes (Tully \& Fisher 1977), and $V_{\mathrm{m}}$ can be determined from the width $W$ of the $21-\mathrm{cm}$ HI line or from the optical rotation curve. This relationship is a powerful and accurate distance indicator for the spiral galaxies and has been extensively used for such a purpose (e.g., Sakai et al. 2000). In the present study it is critically important to use near-infrared wavelength photometry data, since the LV region is close to the Galactic plane, and the effect of Galactic extinction is significantly reduced in nearinfrared wavelengths compared to optical wavelengths.

\subsection{H-band TFR calibration sample}

As a first step, we determine the parameters of the corresponding IRTFR. For such a purpose, we have to use a sample of spiral galaxies having known distances, $H$-band magnitudes $m_{H}$ and rotational velocities $V_{\mathrm{m}}$ measured in the same systems as those of LV sample galaxies. As a matter of fact, one can use two possible samples: either a sample of nearby galaxies having accurate distances measured from Cepheids or TRGB or a larger sample of more remote galaxies, distances of which are determined from their redshifts after correction for attracting various galaxy concentrations. After having tested the two calibration methods, we concluded that the second one gives more secure results, mainly due to the large size of the available sample and because nearby galaxies with large angular dimensions do not have accurate isophotal $H$-band magnitudes in the 2MASS XSC. This calibration method does not give the zero point of the IRTFR, since the absolute magnitudes are computed using the Hubble law, and thus the zero point depends on the Hubble constant. But this is convenient for computing the peculiar velocities of the LV galaxies, as shown in Sect. 4. Hereafter, we use $H_{0}=70 \mathrm{~km} \mathrm{~s}^{-1} \mathrm{Mpc}^{-1}$.

The galaxies of the calibration sample satisfy the same conditions as those of the LV sample (see Sect. 2.1 and below), and their parameters used for the calibration have been computed in the same way as those of the LV sample (see Sect. 4.1 for details of the corrections on radial velocities). Thus no systemactic difference is introduced between the two samples.

The parameters needed for the calibration are the $H$-band magnitude $m_{H}$, the maximum rotational velocity $V_{\mathrm{m}}$, and the distance $D$ for each sample galaxy. Our calibration sample is an all-sky sample of edge-on galaxies with uniform $H$-band photometry and accurate maximum rotational velocities. We use the 2MASS XSC for $m_{H}$ and HyperLEDA (Paturel et al. 2003a) for $V_{\mathrm{m}}$ and recession velocities. We put the following conditions on galaxies to be selected for the calibration sample.

1. Their recession velocities $V_{\mathrm{r}}$ are lower than $8000 \mathrm{~km} \mathrm{~s}^{-1}$, allowing accurate correction for infall in nearby clusters.

2. In order to obtain an accurate distance from $V_{\mathrm{r}}$, we only keep galaxies having uncertainties on $V_{\mathrm{r}}$ less than $100 \mathrm{~km} \mathrm{~s}^{-1}$. Moreover, we reject all the nearby galaxies having $V_{\mathrm{r}}<$ $1000 \mathrm{~km} \mathrm{~s}^{-1}$; indeed, they are generally members of groups, and the internal motions in groups, about $80 \mathrm{~km} \mathrm{~s}^{-1}$ on the line-of-sight, introduce an additional scatter on their distances derived from the redshifts. For the same reason, we eliminate all the galaxies located within the two important clusters of galaxies having $V_{\mathrm{r}}<6000 \mathrm{~km} \mathrm{~s}^{-1}$, namely all the 45 galaxies at less than $15^{\circ}$ and $3^{\circ}$ from the centers of the Virgo and Coma clusters, respectively.

3. The uncertainties on $V_{\mathrm{m}}$ are lower than $20 \mathrm{~km} \mathrm{~s}^{-1}$; indeed, due to the high value of the slope of the IRTFR, those uncertainties are the main source of the observational dispersion of the IRTFR. Thanks to this condition, the dispersion on the IRTFR due to the measurement uncertainties is only 0.14 mag. in absolute magnitude, negligible compared to the intrinsic one ( $\sim .3$ mag, see Sect. 3.2).

4. Similar to the LV galaxies, axial ratios are lower than 0.714 , and the morphological types are between $3(\mathrm{Sb})$ and $8(\mathrm{Sdm})$.

5. H-band magnitudes are taken from the 2MASS XSC. We use $20 \mathrm{mag} / \mathrm{sq}^{\prime \prime}$ isophotal elliptical aperture magnitudes 


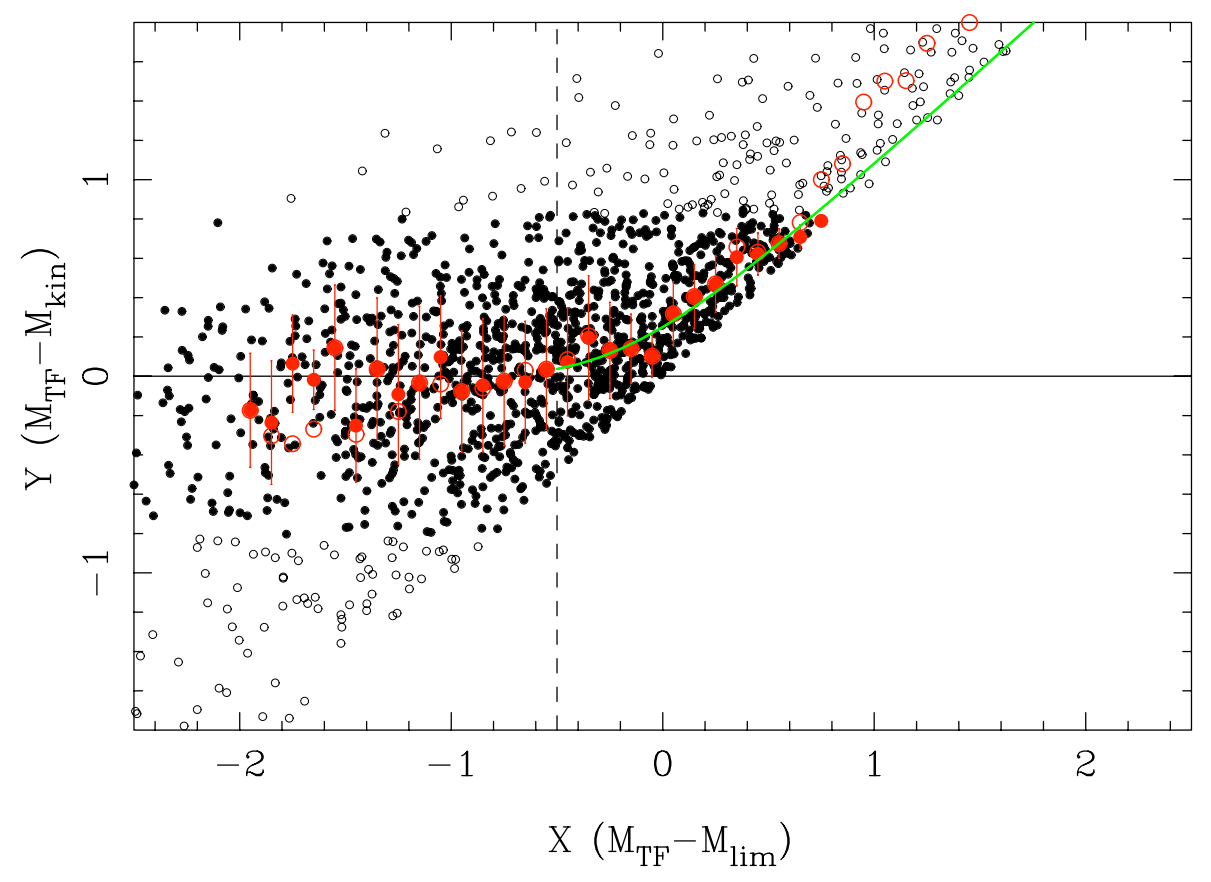

Fig. 4. TFR residuals $\left(Y=M_{\mathrm{TF}}-M_{\text {kin }}\right)$ against the normalized distance modulus $(X)$ for the calibration sample. Small open circles represent the entire sample galaxies, and larger open circles show the average values of $Y$ in $0.1 \mathrm{mag}$. step. Black filled circles are galaxies kept after the removal of outliers in the TFR plot. Larger filled circles with error bars show the average values for them. The vertical dashed line at $X=-0.5$ indicates the upper limit of "bias-free" region. The thick green line shows the analytical curve giving the expected $\langle Y\rangle$ computed from the dispersion of our IRTFR. See text for details. (h_m_i20e) as we did for the LV galaxies. $H$-band apparent magnitudes were corrected for inclination and internal extinction, as well for extinction by our Galaxy. For internal extinction correction, we first derived the amount of extinction in I-band following Tully et al. (1998):

$A_{I}=\left(0.92+1.63\left(\log W_{20}^{\mathrm{c}}-2.5\right)\right) \log (1 / r)$,

where $W_{20}^{\mathrm{c}}$ is the line width at $20 \%$ corrected for inclination in $\mathrm{km} \mathrm{s}^{-1}$ and $r$ is the axis ratio. $A_{I}$ was converted to $H$-band extinction by $A_{H}=0.5 A_{I}$ (Sakai et al. 2000). Amount of galactic extinction toward the direction of each sample galaxy was estimated using the extinction map by Schlegel et al. (1998), and $A_{H} / E(B-V)=0.58$ was assumed.

6. In order to correct the IRTFR for the Malmquist bias, we need our sample to be complete in $m_{H}$ (Theureau et al. 2007). We determine the completeness limit $m_{1}$ by plotting $\log \left[N\left(\leq m_{H}\right)\right]$ versus $m_{H}$, where $N\left(\leq m_{H}\right)$ is the number of sample galaxies with an $H$-band magnitude lower than $m_{H}$. For an homogeneous distribution of galaxies, whatever the luminosity function, the completeness to $m_{1}$ is equivalent to the fact that $\log \left[N\left(\leq m_{H}\right)\right]$ follows the linear relation:

$\log \left[N\left(\leq m_{H}\right)\right]=0.6 m_{H}+C$,

for any $m_{H} \leq m_{\mathrm{lim}}, C$ being a constant. (For $m_{H}>m_{\mathrm{lim}}$, $\log \left[N\left(\leq m_{H}\right)\right]$ increases more slowly than this linear relation.) For our sample, we obtain $m_{\lim }=11.0$.

Thus our final sample comprises all the galaxies figuring both in 2MASS and HyperLEDA and satisfying the conditions 1 to 6 . The number of galaxies in the sample is 1463 .

\subsection{The unbiased IRTFR}

Now we proceed to the determination of the unbiased IRTFR, following the iterative method devised by Theureau et al. (2007). At each iteration, we compute a new IRTFR; the determination of the IRTFR requires the computation of the absolute $H$-band magnitudes of the galaxies, which is carried out from their redshifts, corrected for non-Hubble motions in the same way as those of the galaxies of the LV sample (see Sect. 4.1).
In a first step, we determine the IRTFR from our entire calibration sample, limited however to $M_{H} \leq-22.1$. That cut is made since we found that the slope of the IRTFR changes at this value, being steeper at $M_{H}>-22.1$. Moreover, all the LV galaxies with $m_{H} \leq 11.0$ are in that part of the IRTFR, for which the cut does not introduce any classical Malmquist bias. The bulk of the calibration galaxies (94\%) remains in the sample after this cut. We obtain the best-fit coefficients for the IRTFR:

$M_{\mathrm{TF}}=-8.06 \log V_{\mathrm{m}}-5.31$

with $V_{\mathrm{m}}$ in $\mathrm{km} \mathrm{s}^{-1}$.

This relation is biased since the sample is not complete in a definite interval of absolute magnitudes, but only in apparent magnitudes. For a given sample galaxy, one can determine $M_{\mathrm{TF}}$ from Eq. (7), and also its kinematical absolute $H$-band magnitude $M_{\text {kin }}$ from the corrected redshift. The quantity $Y=$ $M_{\mathrm{TF}}-M_{\text {kin }}$ exhibits the Malmquist bias, through the uncertainties and the intrinsic dispersion of the IRTFR, since $\langle Y\rangle>0$ for our sample (Fig. 4).

On the other hand, Teerikorpi (1975) has shown that the Malmquist bias depends only on the normalized distance modulus $X=M_{\mathrm{TF}}^{\mathrm{c}}-M_{\text {lim }}$, where $M_{\mathrm{TF}}^{\mathrm{c}}$ is the $H$-band absolute magnitude of the galaxy considered as derived from the unbiased IRTFR, and $M_{\text {lim }}$ the $H$-band absolute magnitude cut off corresponding to the limiting apparent magnitude $m_{\mathrm{lim}}$. We compute $M_{\text {lim }}$ using the corrected redshift of the galaxy; for $M_{\mathrm{TF}}^{\mathrm{c}}$, we take the value of $M_{\mathrm{TF}}$ obtained from the biased IRTFR (Eq. (7)) as a first approximation.

The plot $Y(X)$ is shown in Fig. 4; the absence of bias for a given value of $X$ is characterized by $\langle Y(X)\rangle=0$. One can see that in our sample there is a region free of bias, at $X \leq-0.5$; for $X>-0.5$ the bias increases monotonically, reaching about 1 at $X=1$. Thus, in the second iterative step, we keep only the calibration galaxies having $X \leq-0.5$, in the region free of bias, and with those 584 galaxies we obtain

$M_{\mathrm{TF}}=-8.58 \log V_{\mathrm{m}}-4.06$

as a new IRTFR $^{1}$.

${ }^{1}$ Note that Eq. (8) can be written as $M_{\mathrm{TF}}=-8.58 \log V_{\mathrm{m}}-4.06+$ $5 \log \left(H_{0} / 70\right)$ if we take a different value for $H_{0}$. 


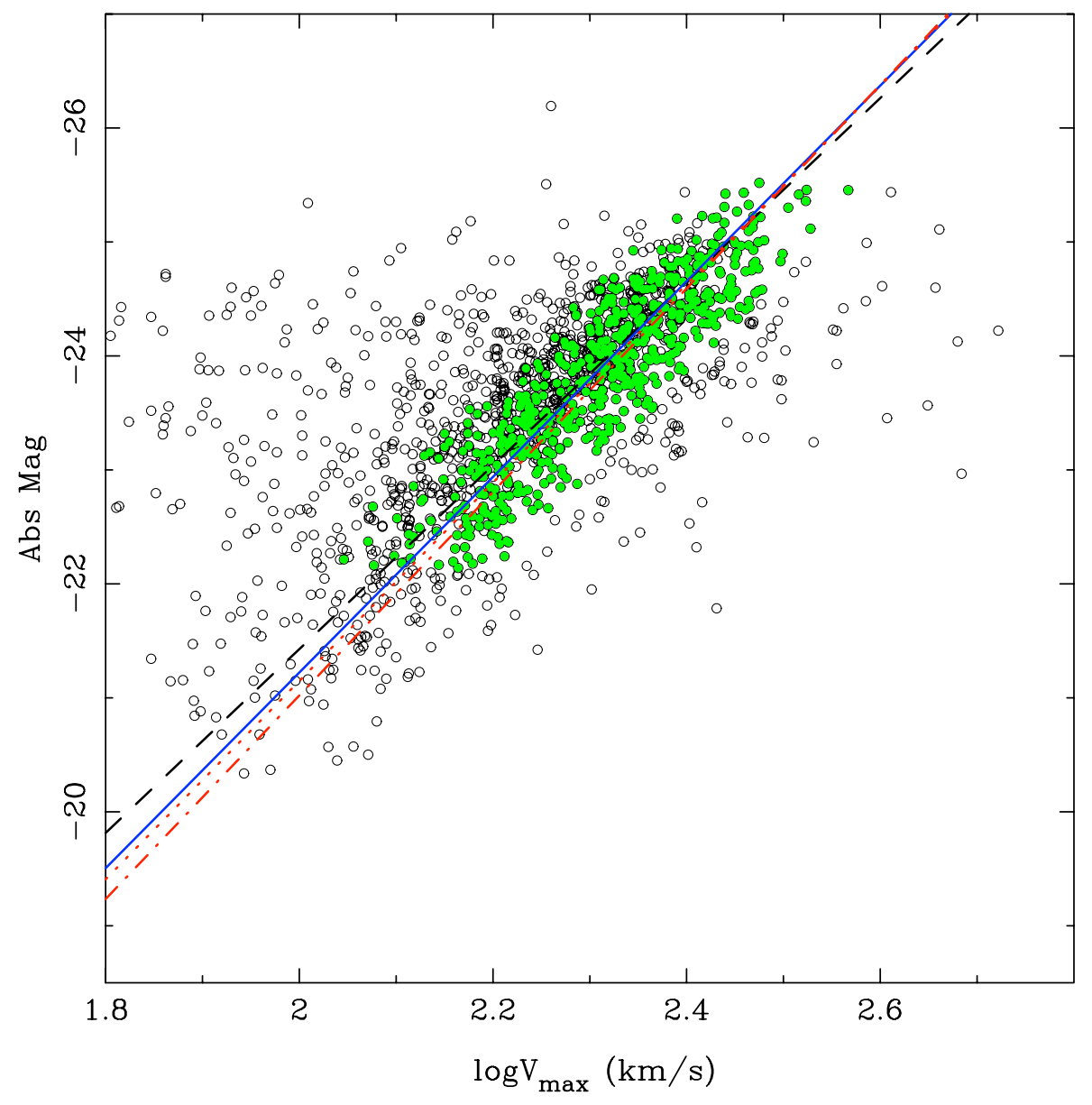

Fig. 5. TFR for the calibration sample. Open circles represent the entire calibration sample galaxies, and filled circles are for the "biasfree" subsample, after removal of outliers in the TFR. The dashed line is the best-fit for the entire sample (Eq. (7)), and the solid line is the best-fit result of the first iteration on the unbiased subsample (Eq. (8)). The dotted line is the result of the second iteration, which is not significantly different from the result of the first iteration. The dot-dashed line displays the TFR by Masters et al. (2008) after conversions in $\mathrm{HI}$ line widths and $H$-band magnitude systems (Eq. (9)).
In Fig. 5 we show this fit as a solid line over the TFR distribution of the calibration sample. However, this IRTFR may not be completely bias free, since the $X$ used was not equal to $M_{\mathrm{TF}}^{\mathrm{c}}-M_{\mathrm{lim}}$, but to $M_{\mathrm{TF}}-M_{\mathrm{lim}}$. So, in a third iterative step, we draw the new plot $(X, Y)$ and select the galaxies in the corresponding region bias-free to compute the next IRTFR. We find that the IRTFR does not change significantly. Thus the unbiased adopted IRTFR is given by Eq. (8). The corresponding average dispersion of $M_{\mathrm{TF}}\left(V_{\mathrm{m}}\right)$ around the relation is $\sigma=0.31$. In Fig. 4 the data points and average values for the sample galaxies after the removal of outliers in the TFR plot are shown as filled circles. Also in Fig. 4 the analytic function of $\langle Y(X)\rangle$ described by Theureau et al. (2007) is plotted for this sample, and it agrees very well with the data.

Note that Masters et al. (2008) have derived an IRTFR in the $H$-band from a sample of 2MASS calibration galaxies carefully chosen, having total extrapolated $H$-band magnitudes $M_{H_{\text {tot }}}$ and accurate HI profile widths $W_{50}$ at $50 \%$ of the peak intensity. After corrections for various statistical biases, they obtain the IRTFR in $H$-band as $M_{H_{\text {tot }}}$ versus the width $W_{50}^{\text {corr }}$ corrected for inclination. Accounting for the relation between $W_{50}$ and $W_{20}$ (Paturel et al. 2003b) and the average difference $M_{H_{\mathrm{tot}}}-M_{H_{\mathrm{iso}}}=$ -0.15 between total $H$-band magnitudes and our isophotal ones, their relation becomes

$M_{H_{\text {iso }}}=-8.93 \log V_{\mathrm{m}}-3.16$.

This relation leads to $M_{H}$ larger than ours by $0.08 \pm 0.05$ mag in the range $2.2 \leq \log V_{\mathrm{m}} \leq 2.5$ corresponding to our $\mathrm{LV}$ galaxies of interest having $m_{H} \leq 11.0$. Thus the agreement is excellent, as that of the scatter of the $M_{\mathrm{TF}}$ at a given $V_{\mathrm{m}}$, which is $0.37 \mathrm{in}$ Masters et al. (2008) compared to our value of 0.31 .

\section{Non-Hubble residual peculiar velocities of the LV sample galaxies}

The non-Hubble residual peculiar velocity $V_{\mathrm{p}}$ of a galaxy is defined by

$V_{\mathrm{p}}=V_{\text {corr }}-H_{0} D$

where $H_{0}$ is the Hubble constant, $D$ the distance of the galaxy computed here from the IRTFR (thus independently of the redshift) and $V_{\text {corr }}$ is the measured radial velocity of the object referred to the centroid of the Local Group (LG) and corrected for the known local non-Hubble motions which include different velocities for the $\mathrm{LG}$ and the galaxy considered. We will use the IRTFR in Eq. (8). $H_{0} D$ does not depend on the value of $H_{0}$ adopted if $H_{0}$ is the same as the one used for the determination of the IRTFR (in this case $70 \mathrm{~km} \mathrm{~s}^{-1} \mathrm{Mpc}^{-1}$ ).

\subsection{Computation of $V_{\text {corr }}$}

To obtain $V_{\text {corr }}$, first we convert the measured heliocentric radial velocities $V_{\mathrm{h}}$ to radial velocities $V_{\mathrm{LG}}$ referred to the centroid of the LG using the equation of Tully et al. (2008):

$V_{\mathrm{LG}}=V_{\mathrm{h}}+305 \sin l \cos b-86 \cos l \cos b-33 \sin b$.

Then the known local non-Hubble motions we have to correct $V_{\mathrm{LG}}$ for are the following:

(1) The repulsion of the LG from the center of the LV, recently evidenced convincingly by Tully et al. (2008) thanks to very accurate distance measurements of galaxies located at less 
than $10 \mathrm{Mpc}$ from us, based on the measurements of apparent brightness of TRGB stars. The repulsion velocity of the structure at the boundary of the LV (named the "Local Sheet", which includes the LG) is reported to be $259 \mathrm{~km} \mathrm{~s}^{-1}$ toward $\ell=210^{\circ}, b=-2^{\circ}$. We are looking at a similar repulsion for our sample galaxies. Those objects and the LG are located nearly at opposite borders of the LV, and we need to correct the radial velocity of sample galaxies for this LG motion against the LV. By including the motion of the centroid of the LG within the Local Sheet $\left(66 \mathrm{~km} \mathrm{~s}^{-1}\right.$ towards $\ell=11^{\circ}, b=22^{\circ}$; Tully et al. 2008) $)^{2}$, one finds that the centroid of the LG has a velocity of $202 \mathrm{~km} \mathrm{~s}^{-1}$ toward $\ell=215^{\circ}, b=5^{\circ}$ with respect to the LV. This results in corrections of $\sim-180 \mathrm{~km} \mathrm{~s}^{-1}$ for radial velocities of our sample galaxies.

(2) The infalls towards three nearby mass concentrations, namely the Virgo cluster, the Great Attractor (GA) and the Shapley supercluster. Such corrections are necessary since the infall velocities are quite different for the sample galaxies and for the LG. The infall corrections have been carried out following Mould et al. (2000). In brief, those authors use a simple multi-attractor model; they assume the flows to be independent, thus the respective velocity infalls add to each other. The infall velocity $V_{f}$ towards each attractor at the level of the LG is known. If one assumes that the attractor has the spherical symmetry with a density profile $\rho(r) \propto r^{-\gamma}$, then the infall velocity $V(r)$ is: $V(r) \propto r^{1-\gamma}$, and one can compute the projected velocity component $V_{\text {inf }}$ on the lineof-sight of the galaxy oriented towards the galaxy, of the infall velocity of the object caused by the attractor, as seen from the infalling LG, namely,

$V_{\mathrm{inf}}=V_{f}\left(\frac{r_{\mathrm{a}} \cos \theta-r_{0}}{r_{0 \mathrm{a}}}\right)\left(\frac{r_{0 \mathrm{a}}}{r_{\mathrm{a}}}\right)^{1-\gamma}-V_{f} \cos \theta$,

where $\theta$ is the angle between the directions of the galaxy and of the attractor as seen from us, $r_{0}$ is the distance of the object, $r_{\mathrm{a}}$ is the distance of the attractor, and $r_{0 \mathrm{a}}$ is the distance between the galaxy and the attractor: $r_{0 \mathrm{a}}=$ $\sqrt{r_{0}^{2}+r_{\mathrm{a}}^{2}-2 r_{0} r_{\mathrm{a}} \cos \theta}$.

Following Mould et al. (2000), we take $\gamma=2$, which fits the Virgo cluster. Note that in Eq. (12), the two successive terms represent the difference of the respective projections, on the lineof-sight of the galaxy, of the object and of the LG infall velocities towards the attractor.

Coordinates of the attractors and $V_{f}$ values are taken from Mould et al. (2000); we have adopted for $r_{\mathrm{a}}: 17.4 \mathrm{Mpc}, 65.8 \mathrm{Mpc}$ and 190.3 Mpc for Virgo, GA and Shapley supercluster, respectively, corresponding to $H_{0}=70 \mathrm{~km} \mathrm{~s}^{-1} \mathrm{Mpc}^{-1}$ for velocities of the attractors corrected for infalls. The distances $r_{0}$ of the LV galaxies have been computed from their redshifts referred to the centroid of the LG.

The values of $V_{\text {inf }}$ for our galaxies are on the order of $50 \mathrm{~km} \mathrm{~s}^{-1} \mathrm{Mpc}^{-1}, 200 \mathrm{~km} \mathrm{~s}^{-1} \mathrm{Mpc}^{-1}$, and $20 \mathrm{~km} \mathrm{~s}^{-1} \mathrm{Mpc}^{-1}$ for Virgo, GA, and Shapley infalls, respectively. Taking the repulsion of the LG from the center of the LV into account, the total corrections to $V_{\mathrm{LG}}, \delta V_{\mathrm{LG}}=\delta V_{\mathrm{LV}}-V_{\text {inf }}$ are in fact quite small, about a few tens of $\mathrm{km} \mathrm{s}^{-1}$. If the infall corrections for these nearby mass concentrations are not applied, the peculiar velocities for most of the galaxies in the final sample are

\footnotetext{
2 There is an error in the calculation of the galactic longitude of this motion in Tully et al. (2008) (i.e., $\ell$ of $V_{\mathrm{LS}}^{\mathrm{LG}}$ ) in their Table 3 .
}

100-300 $\mathrm{km} \mathrm{s}^{-1}$ smaller than when using these corrections. Those velocities are near the expected LV expansion, thus infall corrections have to be applied here.

The corrected velocities are given in Table 4.

\subsection{Distances of the LV galaxies}

We compute the distances of the LV galaxies using the IRTFR corrected for Malmquist bias (determined in Sect. 3.2). In order not to introduce any other bias, we have to treat our LV sample exactly as the calibration sample, in particular to limit it to $m_{H} \leq 11.0$ and $M_{H} \leq-22.1$, and also compute $V_{\mathrm{m}}$ the same way. Only 15 galaxies among the 36 of our LV sample have $m_{H} \leq 11.0$, and it happens that all those 15 are in the part $\log V_{\mathrm{m}} \geq 2.10$, which corresponds to $M_{H} \leq-22.1$.

\subsubsection{Determination of the maximum rotational velocity $V_{\mathrm{m}}$}

The HyperLEDA extragalactic database provides the maximum rotational velocity $V_{\mathrm{m}}$ for a number of galaxies. We have used these $V_{\mathrm{m}}$ values for those eight galaxies in our LV sample for which we took HyperLEDA 21-cm data. For the other LV galaxies, $V_{\mathrm{m}}$ was determined using the tight correlation between $V_{\mathrm{m}}$ and $W_{20}^{\mathrm{c}}$ obtained from the calibration galaxies, namely,

$\log V_{\mathrm{m}}=1.12 \log W_{20}^{\mathrm{c}}-0.67$,

where $W_{20}^{c}$ is obtained from the HI profile width at $20 \%$ of the peak value corrected for resolution by

$W_{20}^{\mathrm{c}}=W_{20} / \sin i(1+z)$,

where $i$ is the inclination of the galaxy and $z$ the redshift. Taking the apparent axis values obtained with super-coadded image in 2MASS using $J, H$ and $K$-bands, we derive $i$ from the apparent axis ratio $r$ by

$\cos i=\sqrt{\frac{r^{2}-r_{0}^{2}}{1-r_{0}^{2}}}$,

where $r_{0}$ is the intrinsic minor to major axis ratio for spiral galaxies. Following many previous studies on TFR (e.g., Tully \& Fisher 1977; Sakai et al. 2000; Masters et al. 2008), we take $r_{0}=0.2$.

\subsubsection{Computation of the distances and correction for Malmquist bias}

The distance modulus $\mu$ of any galaxy of our LV sample is given by

$\mu=m_{H}^{\mathrm{c}}-M_{H}^{\mathrm{c}}$,

where $m_{H}^{\mathrm{c}}$ and $M_{H}^{\mathrm{c}}$ are the apparent and absolute $H$-band magnitudes corrected for galactic and internal extinction, respectively. In Table 4 we show the distances for the 15 galaxies with $m_{H}<11.0$. The photometric data are either from our own observations or from 2MASS XSC when our data are not available, as shown in Table 3 . We also show the case where only 2MASS XSC photometry is used. Using only 2MASS photometry eliminates a possibility of systematic difference between data based on different facilities, but since the LV is located at low Galactic latitudes, the 2MASS photometry would suffer from contamination by foreground stars. In our own photometry we carefully removed foreground stars (Sect. 2.4), so this effect should be alleviated. 
Table 4. Distances and peculiar velocities for the "unbiased" Local void sample galaxies.

\begin{tabular}{|c|c|c|c|c|c|c|c|c|c|}
\hline \multirow[b]{2}{*}{ Name } & \multirow[b]{2}{*}{$V_{\text {corr }}^{c}$} & \multicolumn{4}{|c|}{ UH88/IRSF/2MASS ${ }^{a}$} & \multicolumn{4}{|c|}{2 MASS $^{b}$} \\
\hline & & $D^{d}$ & $V_{\mathrm{p}}$ & $D^{* e}$ & $V_{\mathrm{p}}^{* f}$ & $D^{d}$ & $V_{\mathrm{p}}$ & $D^{* e}$ & $V_{\mathrm{p}}^{* f}$ \\
\hline UGC 11246 & 4287 & $83.19_{-12.09}^{+14.15}$ & $-1535_{-990}^{+846}$ & - & - & $82.01_{-11.89}^{+13.91}$ & $-1452_{-889}^{+916}$ & - & - \\
\hline UGC 11301 & 4715 & $62.65_{-8.80}^{+10.24}$ & $331_{-717}^{+616}$ & - & - & $62.10_{-8.72}^{+10.15}$ & $369_{-673}^{+649}$ & - & - \\
\hline NGC 6674 & 3656 & $55.00_{-8.28}^{+9.70}$ & $-194_{-682}^{+580}$ & - & - & $55.00_{-8.28}^{+9.72}$ & $-194_{-682}^{+580}$ & - & - \\
\hline UGC 11320 & 5018 & $73.14_{-9.83}^{+11.39}$ & $-101_{-812}^{+701}$ & 71.80 & $-8_{-797}^{+688}$ & $72.60_{-9.75}^{+11.28}$ & $-63_{-805}^{+695}$ & 71.28 & $30_{-753}^{+720}$ \\
\hline UGC 11344 & 4040 & $65.97_{-9.58}^{+11.21}$ & $-578_{-785}^{+671}$ & - & - & $61.03_{-8.86}^{+10.36}$ & $-227_{-375}^{+971}$ & - & - \\
\hline IRAS $18421+1218$ & 4850 & $75.56_{-14.17}^{+17.72}$ & $-439_{-1327}^{+1061}$ & 70.62 & $-93_{-1240}^{+992}$ & $75.56_{-14.17}^{+17.72}$ & $-439_{-1327}^{+1061}$ & 70.62 & $-93_{-1240}^{+992}$ \\
\hline NGC 6700 & 4774 & $81.73_{-12.23}^{+14.31}$ & $-946_{-1007}^{+856}$ & - & - & $81.73_{-12.23}^{+14.17}$ & $-946_{-1007}^{+857}$ & - & - \\
\hline UGC 11355 & 4555 & $73.17_{-14.14}^{+17.53}$ & $\begin{array}{l}-1066_{-1227}^{+990} \\
-5\end{array}$ & - & - & $73.17_{-14.14}^{+17.53}$ & $\begin{array}{l}-1066_{-1227}^{+991} \\
-5\end{array}$ & - & - \\
\hline IRAS $18575+1845$ & 4859 & $79.60_{-12.22}^{+14.14}$ & $-713_{-1011}^{+855}$ & - & - & $70.26_{-10.84}^{+12.82}$ & $-50_{-234}^{+1422}$ & - & - \\
\hline UGC 11426 & 4620 & $65.12_{-8.93}^{+10.80}$ & $\begin{array}{r}-103_{-953}^{+788} \\
\end{array}$ & 51.66 & $1005_{-756}^{+625}$ & $65.12_{-8.93}^{-10.80}$ & $63_{-953}^{+788}$ & 51.66 & $1005_{-756}^{+625}$ \\
\hline UGC 11568 & 4288 & $57.01_{-8.11}^{+9.46}$ & $298_{-662}^{+568}$ & - & - & $57.01_{-8.11}^{+9.46}$ & $298_{-662}^{+567}$ & - & - \\
\hline MCG -02-52-006 & 3990 & $56.23_{-10.17}^{+12.54}$ & $55_{-914}^{-602}$ & 53.97 & $213_{-877}^{+712}$ & $56.23_{-10.17}^{+12.54}$ & $55_{-914}^{+762}$ & 53.97 & $213_{-878}^{+712}$ \\
\hline MCG -01-52-016 & 3953 & $42.05_{-5.93}^{+6.99}$ & $1009_{-525}^{+446}$ & 39.17 & $1211_{-489}^{+415}$ & $42.05_{-5.93}^{+6.99}$ & $1009_{-525}^{+414}$ & 39.17 & $1211_{-490}^{+415}$ \\
\hline UGC 11961 & 4223 & $64.00_{-9.49}^{+11.20}$ & $-256_{-807}^{+684}$ & 62.20 & $-130_{-784}^{+665}$ & $64.00_{-9.49}^{+11.20}$ & $-256_{-807}^{+684}$ & 62.20 & $-130_{-784}^{+665}$ \\
\hline NGC 7264 & 4231 & $82.58_{-13.94}^{+16.77}$ & $\begin{array}{l}-80 / \\
-1549_{-1174}^{+976}\end{array}$ & - & - & $82.58_{-13.94}^{+16.77}$ & $-1549_{-1173}^{+976}$ & - & - \\
\hline
\end{tabular}

Notes. ${ }^{(a)}$ These values are based on $H$-band photometry from UH88 and IRSF observations when they are available and on 2MASS XSC otherwise. ${ }^{(b)}$ These values are based on $H$-band photometry from 2 MASS XSC alone. ${ }^{(c)}$ See Sect. 4.1. ${ }^{(d)}$ Distances from IRTFR with bias corrections (when its normalized distance moduli $X>-0.5$; see Sect. 4.2.2 for details). ${ }^{(e)}$ Distances before the correction for bias for galaxies with $X>-0.5$. (f) Peculiar velocity without correction for bias (i.e., using $D^{*}$ instead of $D$ ).

As explained above (Sect. 3.2), $M_{H}^{\mathrm{c}}$ is determined from the value of the absolute magnitude $M_{\mathrm{TF}}\left(V_{\mathrm{m}}\right)$ given by the IRTFR free of Malmquist bias. However, there is a bias in the LV sample since it is limited in apparent magnitude. For a given $V_{\mathrm{m}}$, the less luminous galaxies are not included in the sample, and this causes the average absolute magnitude $\langle M\rangle$ at $V_{\mathrm{m}}$ to be biased toward being more luminous than the true value. This effect is shown in Fig. 4 for the case of the calibration sample. In this figure, $X$ is a normalized distance modulus $M_{\mathrm{TF}}-M_{\mathrm{lim}}$ where $M_{\text {lim }}=m_{\text {lim }}-\mu_{\text {kin }}$ is an absolute magnitude cut off, $\mu_{\text {kin }}$ the distance modulus computed from the radial velocity, and $Y$ a normalized magnitude $M_{\mathrm{TF}}-M_{\text {kin }}$, where $M_{\text {kin }}$ is the absolute magnitude corresponding to $m_{H}: M_{\mathrm{kin}}=m_{H}-\mu_{\mathrm{kin}}$. As discussed in Sect. 3.2, there is a bias in $\langle Y\rangle$ at $X>-0.5$, and thus in order to use galaxies with $X>-0.5$ to derive the average peculiar velocity, we need to correct the bias by applying the correction to the absolute magnitude obtained from IRTFR: $M_{H}^{\mathrm{c}}(X)=M_{\mathrm{TF}}\left(V_{\mathrm{m}}\right)-\langle Y(X)\rangle$. The value of $\langle Y(X)\rangle$ is computed analytically from the TF dispersion, following Theureau et al. (2007).

Among the 15 galaxies with $m_{H} \leq 11.0$, there are six galaxies with $X>-0.5$. Correction factors for the distances are less than $8 \%$, except for UGC 11426 where it is $26 \%$.

\subsection{Residual peculiar velocities of the LV galaxies}

We compute the residual peculiar velocities $V_{\mathrm{p}}$ with Eq. (10), using the distance $D$ determined in the previous section. "Biascorrected" distances are used for the galaxies with $X>-0.5$. The calculated $V_{\mathrm{p}}$ are shown in Table 4 . In the results based on UH88/IRSF/2MASS photometry, 10 galaxies have negative values of $V_{\mathrm{p}}$, five have positive ones. In Fig. 6 we show the distribution of $V_{\mathrm{p}}$ against the radial velocities $V_{\text {corr }}$. There appears to be no significant correlation between the radial velocities and the peculiar velocities.

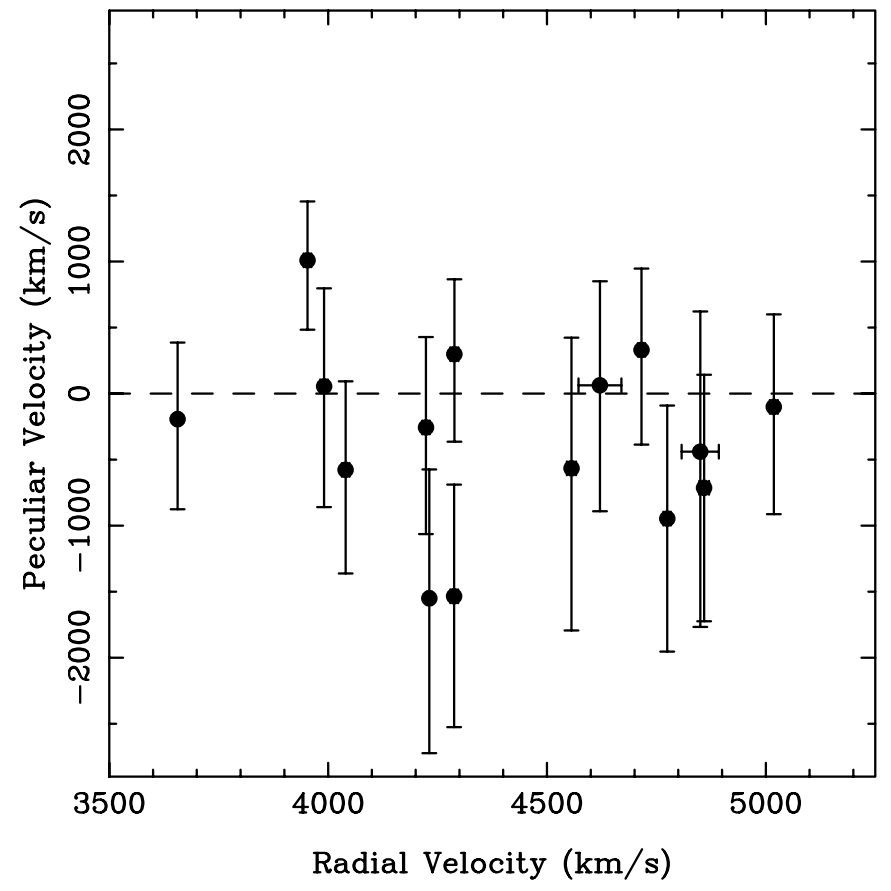

Fig. 6. Corrected radial velocities and peculiar velocities of the "unbiased" Local Void sample galaxies. For galaxies with the normalized distance modulus $X=M_{\mathrm{TF}}-M_{\lim }>-0.5$, the bias corrections were applied (see text for details).

Accounting for the uncertainties on each $V_{\mathrm{p}}$ as listed in Table 4 , we obtain the average value of $V_{\mathrm{p}}:\left\langle V_{\mathrm{p}}\right\rangle$ is $-419+$ $208-251 \mathrm{~km} \mathrm{~s}^{-1}$ for the case with UH88/IRSF/2MASS photometry, and $\left\langle V_{\mathrm{p}}\right\rangle$ is $-319+204-246 \mathrm{~km} \mathrm{~s}^{-1}$ for the case with 2MASS XSC photometry alone. Thus $\left\langle V_{\mathrm{p}}\right\rangle$ is not significantly different from a zero value. The values within a $3 \sigma$ error 
range from $-1172 \mathrm{~km} \mathrm{~s}^{-1}$ to $205 \mathrm{~km} \mathrm{~s}^{-1}$ for UH88/IRSF/2MASS photometry. If the LV has a general expansion, one may think that the expansion at the far boundary of the LV we investigate is comparable to that of the Local Sheet, i.e., $259 \mathrm{~km} \mathrm{~s}^{-1}$ (Tully et al. 2008), and it is slightly out of but very close to the $3 \sigma$ error range of our results. Due to the size of the error, we cannot evidence such an expansion of the far-side boundary of the LV. The size of the errors in our peculiar velocities comes mainly from the intrinsic scatter of the IRTFR ( $\sigma=0.31$ in absolute magnitude). There are some other sources, such as the uncertainties on the widths of the HI line profiles, uncertainty in $H$-band photometry, and in radial velocities, but they are less than the effect of the scatter of the TFR.

One can note that the dispersion of the $V_{\mathrm{p}}$ around their average is also about $200 \mathrm{~km} \mathrm{~s}^{-1}$, as for the expected uncertainty, which shows that there is no systematic variations in $V_{\mathrm{p}}$ among our sample galaxies.

\section{Summary and conclusions}

The LV, the nearest void of galaxy distribution from us, is expected to undergo a general expansion, as in any void of galaxies, due to the lack of matter within it. Tully et al. (2008) show that the Local Group and galaxies near the Local Group, i.e., the Local Sheet at the edge of the LV, move away from the center of the LV with a velocity of $259 \mathrm{~km} \mathrm{~s}^{-1}$.

In the present study, we investigated the peculiar velocities of the galaxies located at the opposite edge of the LV with respect to the Local Group to see if they show an expansion from the center of the LV, by using the IR Tully-Fisher relation to compute their distances. The sample galaxies have an edge-on spiral morphology and radial velocities between $3000 \mathrm{~km} \mathrm{~s}^{-1}$ and $5000 \mathrm{~km} \mathrm{~s}^{-1}$. Gathering 19 HI line width measurements by ourselves and those in the literature leads to a final number of sample galaxies of 36. We also made $H$-band photometry for the majority of the sample galaxies. To derive the IR Tully-Fisher relation, we used a large sample of galaxies having maximum rotational velocities in HyperLEDA (Paturel et al. 2003a) and $H$-band isophotal magnitudes in 2MASS XSC, complete to $m_{H}=11.0$. The IRTFR free from the Malmquist bias was obtained from that sample, and then was used to compute the distances of the 15 LV galaxies having $m_{H} \leq 11.0$. After the corrections for the infall motions toward the nearby clusters/concentrations and the motion of the Local Group away from the center of the LV, the residual peculiar velocities $V_{\mathrm{p}}$ for the $15 \mathrm{LV}$ galaxies have been obtained. The average value after the correction for the Malmquist bias (which is thought to affect $V_{\mathrm{p}}$ of some of the sample galaxies) is $\left\langle V_{\mathrm{p}}\right\rangle=-419_{-251}^{+208} \mathrm{~km} \mathrm{~s}^{-1}$. This is not significantly different from zero, and it does not reject the possibility that these galaxies have a motion against the LV equivalent to that of the Local Sheet $\left(259 \mathrm{~km} \mathrm{~s}^{-1}\right)$.

Padilla et al. (2005) made a $\Lambda$ CDM numerical simulation on the properties of dark matter halos and galaxies around voids and find a linear relation between the maximum outflow velocity $v_{\max }$ and the distance from the center of the void $r_{\text {void }}: v_{\max }=v_{0} r_{\text {void }}$, where the best-fit value of $v_{0}$ is $14.5 \mathrm{~km} \mathrm{~s}^{-1} h \mathrm{Mpc}^{-1}$. If we adopt the radius of the void to be $2500 \mathrm{~km} \mathrm{~s}^{-1}$ and $h=0.7$, this gives the $v_{\max }$ of $360 \mathrm{~km} \mathrm{~s}^{-1}$. Such a value is significantly higher than the motion of $259 \pm 25 \mathrm{~km} \mathrm{~s}^{-1}$ of the Local Sheet away from the $\mathrm{LV}$, and at $3.7 \sigma$ from our $\left\langle V_{\mathrm{p}}^{\mathrm{c}}\right\rangle$. Thus it does not seem to account correctly for the expansion of the LV. However, the geometry of the LV is more complex than a simple sphere, consisting in a void within two large voids (Tully et al. 2008). Thus the maximum velocity Padilla et al. (2005) measured in their simulated voids might not be fully appropriate for the comparison with the expansion velocity of the LV.

Finally, the uncertainty $\sim 200 \mathrm{~km} \mathrm{~s}^{-1}$ of our $\left\langle V_{\mathrm{p}}^{\mathrm{c}}\right\rangle$ is not sufficient for proving the expansion found by Tully et al. (2008) for the Local Sheet. Smaller uncertainty would be achieved if we were able to use more galaxies - i.e., using galaxies with apparent magnitude fainter than $m_{H}=11.0$. However, since the number of galaxies at the far-side of the LV, which can be used for IRTFR, would not exceed $\sim 50$, we may need an alternative, more accurate distance estimator to conclusively know whether the opposite edge of the LV undergoes an expansion.

Acknowledgements. We thank staff members of the telescope facilities used in this work (Okayama Astrophysical Observatory, Nançay radiotelescope, the Infrared Survey Facility, and the University of Hawaii 2.2 m telescope) for their support during observations. We would like to thank the referee (B. Tully) for helpful comments that improved the paper. II is grateful to M. Saitō who raised the initial idea of this work, A. T. Roman for his participation in the observations in Okayama, and K. Nakanishi and K. Ohta for supporting observations and giving thoughtful suggestions. II was supported by a Research Fellowship of the Japan Society for the Promotion of Science (JSPS) for Young Scientists during parts of this research.

\section{References}

Bottinelli, L., Gouguenheim, L., Fouqué, P., \& Paturel, G. 1990, A\&AS, 82, 391 Burstein, D. 2000, in Cosmic Flows Workshop, ed. S. Courteau, \& J. Willick, ASP Conf. Ser., 201, 178

Ceccarelli, L., Padilla, N. D., Valotto, C., \& Lambas, D. G. 2006, MNRAS, 373, 1440

Faber, S. M., \& Burstein, D. 1988, Motions of galaxies in the neighborhood of the local group, ed. V. C. Rubin, \& G. V Coyne, 115

Fouqué, P., Durand, N., Bottinelli, L., Gouguenheim, L., \& Paturel, G. 1990, A\&AS, 86, 473

Hewitt, J. N., Haynes, M. P., \& Giovanelli, R. 1983, AJ, 88, 272

Masters, K. L., Springob, C. M., \& Huchra, J. P. 2008, AJ, 135, 1738

Mould, J. R., Huchra, J. P., Freedman, W. L., et al. 2000, ApJ, 529, 786

Nagashima, C., Nagayama, T., Nakajima, Y., et al. 1999, in Star Formation 1999, Proc. Star Formation 1999, held in Nagoya, Japan, June 21-25, ed. T. Nakamoto, Nobeyama Radio Observatory, 397

Nagayama, T., Nagashima, C., Nakajima, Y., et al. 2003, in Instrument Design and Performance for Optical/Infrared Ground-based Telescopes, ed. M. Iye, \& A. F. M. Moorwood, Proc. SPIE, 4841, 459

Nakanishi, K., Takata, T., Yamada, T., et al. 1997, ApJS, 112, 245

Nilson, P. 1973, Uppsala general catalogue of galaxies, ed. P. Nilson, Uppsala Astronomiska Observatoriums Annaler

Padilla, N. D., Ceccarelli, L., \& Lambas, D. G. 2005, MNRAS, 363, 977

Paturel, G., Petit, C., Prugniel, P., et al. 2003a, A\&A, 412, 45

Paturel, G., Theureau, G., Bottinelli, L., et al. 2003b, A\&A, 412, 57

Roman, A. T., Iwata, I., \& Saitō, M. 2000, ApJS, 127, 27

Sakai, S., Mould, J. R., Hughes, S. M. G., et al. 2000, ApJ, 529, 698

Saunders, W., Sutherland, W. J., Maddox, S. J., et al. 2000, MNRAS, 317, 55

Schlegel, D. J., Finkbeiner, D. P., \& Davis, M. 1998, ApJ, 500, 525

Sheth, R. K., \& van de Weygaert, R. 2004, MNRAS, 350, 517

Skrutskie, M. F., Cutri, R. M., Stiening, R., et al. 2006, AJ, 131, 1163

Springob, C. M., Haynes, M. P., Giovanelli, R., \& Kent, B. R. 2005, ApJS, 160, 149

Teerikorpi, P. 1975, A\&A, 45, 117

Theureau, G., Hanski, M. O., Coudreau, N., Hallet, N., \& Martin, J.-M. 2007, A\&A, 465, 71

Tully, R. B., \& Fisher, J. R. 1977, A\&A, 54, 661

Tully, R. B., \& Fisher, J. R. 1987, Nearby Galaxies Atlas, ed. R. B. Tully, \& J. R. Fisher (Cambridge University Press)

Tully, R. B., Pierce, M. J., Huang, J., et al. 1998, AJ, 115, 2264

Tully, R. B., Shaya, E. J., Karachentsev, I. D., et al. 2008, ApJ, 676, 184 